\title{
Validación de un diseño fotovoltaico con un consumo de 30 Watts DC, para ser utilizado con un sistema NFT en comunidades sin acceso a la red eléctrica
}

\section{Validation of a photovoltaic design with a consumption of 30 Watts DC, to be used with an NFT system in communities without access to the electricity grid}

\author{
Gabriel López ${ }^{l}$, José Jirón ${ }^{l}$, Karina Rodríguez, ${ }^{l}$, Sergio Valdés ${ }^{l}$, Héctor Rosales ${ }^{{ }^{*}}$ \\ ${ }^{1}$ Licenciatura en Sistemas Eléctricos y Automatización-Centro Regional Universitario de Coclé-Universidad de Panamá
}

\begin{abstract}
Resumen Este trabajo se centró en el dimensionamiento de un sistema solar fotovoltaico y su implementación en un sistema hidropónico de tipo Nutrient Film Technique (NFT). El sistema solar se especificó para tener una autonomía en energía de dos días. Para lograr este objetivo, se consideró el uso de una bomba DC de 20W para la recirculación de los nutrientes, durante dos periodos de dos horas cada uno y también se utilizó un bombillo de $10 \mathrm{~W}$ durante dos horas. Con estos requerimientos se pudieron obtener las especificaciones del panel solar, la batería y el controlador de carga. De acuerdo con los resultados obtenidos, se pudo demostrar que este tipo de sistemas hidropónico se puede implementar con un sistema solar fotovoltaico de baja capacidad y con un bajo presupuesto, muy adecuado para comunidades de escasos recursos y sin acceso a la red eléctrica pública.
\end{abstract}

Palabras clave Sistema solar fotovoltaico, hidroponía, sistema NFT.

Abstract This work focused on the sizing of a solar photovoltaic system and its implementation in a NFT hydroponic system. The solar system was specified to have an autonomy in energy of two days. To achieve this goal, the use of a 20W DC pump for the recirculation of nutrients was considered, for two periods of two hours each and a $10 \mathrm{~W}$ bulb was also used for two hours. With these requirements it was possible to obtain the specifications of the solar panel, the battery and the charge controller. According to the results obtained, it was possible to demonstrate that this type of hydroponic systems can be implemented with a low capacity photovoltaic solar system with a low budget, very suitable for communities with limited resources and without access to the public electricity grid.

Keywords Solar system, photovoltaic, hydroponics, NFT system.

* Corresponding author: sergiovaldes908@gmail.com

\section{Introducción}

La hidroponía es una técnica de producción agrícola en la que se cultiva sin suelo y donde los elementos nutritivos son entregados a las plantas mediante una solución. Por medio de las raíces las plantas reciben la solución nutritiva disuelta en agua con todos los elementos químicos necesarios para el desarrollo de la planta.

Los cultivos sin suelo se centran en eliminar el vertido de los lixiviados y con ello evitar la contaminación de los suelos y los acuíferos. También, estos sistemas contribuyen al ahorro de agua en zonas semiáridas y con escasez de recursos hídricos, al reutilizar toda o parte del agua lixiviada [1].
Un método popular de hidroponía es el sistema tipo NFT (del inglés, Nutrient Film Technique). Este tipo de sistema hace recircular una capa fina de solución nutritiva de manera continua o intermitente; a través de unos canales de tubos PVC, polietileno o poliuretano con forma rectangular o circular, escalonada, en zigzag o vertical, llamados canales de cultivo [2].

Para implementar los sistemas hidropónicos tipo NFT, se requiere de algún tipo de energía para su funcionamiento. El uso de energías renovables es una opción que cada día tiene más beneficios y muchas aplicaciones. Una de las fuentes de 
energías renovable con la que contamos, es la energía solar fotovoltaica que consiste en convertir directamente la radiación solar en electricidad.

En el trabajo realizado por [3], se implementa un sistema para el cultivo hidropónico de 648 plantones de lechuga, utilizando la energía solar fotovoltaica. El sistema solar fue especificado para trabajar en corriente alterna, pues debía alimentar una bomba centrífuga de 120 VAC de 1/4 HP. Este proyecto tuvo un total de costos directos de USD 2,694.62, de los cuales USD 1,504.73 fueron en concepto de equipos y materiales para la implementación del sistema solar fotovoltaico.

Para una familia promedio, implementar un sistema hidropónico de esta magnitud, puede resultar casi imposible; pero si se realizan adecuaciones al diseño tanto del sistema hidropónico como del sistema solar fotovoltaico, se pueden reducir los costos directos de inversión. Para un sistema doméstico se pueden utilizar materiales reciclados para disminuir los costos de inversión en el sistema. Es importante señalar que en la República de Panamá existe un aproximado de 100,000 familias que no cuentan con servicio de energía eléctrica en sus hogares [4], por lo que este tipo de proyectos puede representar una alternativa para el cultivo de nuevos productos para consumo familiar.

La finalidad de este trabajo consiste en dimensionar un sistema solar fotovoltaico diseñado a la medida de un sistema hidropónico tipo NFT. Esto permitirá energizar, eficientemente, una bomba de agua de 20 W DC y así, hacer recircular una solución de nutrientes en un sistema hidropónico, que puede estar ubicado en comunidades de escasos recursos y sin acceso al servicio eléctrico.

\section{Metodología}

\subsection{Dimensionamiento del sistema}

El dimensionamiento del sistema solar fotovoltaico está basado en el método presentado por [5]. Este método muestra los valores estimados de consumo de los equipos y la carga total del sistema.

Tabla 1. Especificaciones de la carga y el tiempo de uso

\begin{tabular}{|c|c|c|c|c|c|c|}
\hline \multicolumn{7}{|c|}{ Especificaciones de la carga y el tiempo de uso } \\
\hline Tipo & Equipos & $\begin{array}{c}\text { Canti- } \\
\text { dad }\end{array}$ & $\begin{array}{l}\text { Potencia } \\
\text { Nominal }\end{array}$ & $\begin{array}{l}\text { Sub- } \\
\text { total }\end{array}$ & $\begin{array}{c}\text { Horas } \\
\text { diarias }\end{array}$ & WH/día \\
\hline \multirow{6}{*}{$\begin{array}{c}\text { Sistema } \\
\text { Solar } \\
\text { Foto- } \\
\text { voltaico }\end{array}$} & $\begin{array}{l}\text { Bomba } \\
\text { de agua }\end{array}$ & 1 & $20 \mathrm{~W}$ & $\begin{array}{l}20 \\
W\end{array}$ & 4 & 80 \\
\hline & Bombillo & 2 & $5 \mathrm{~W}$ & $\begin{array}{l}10 \\
W\end{array}$ & 2 & 20 \\
\hline & \multicolumn{5}{|c|}{ Subtotal (WH/día) } & 100 \\
\hline & \multicolumn{5}{|c|}{$15 \%$ de pérdida $=(100 * 0.15)$} & 15 \\
\hline & \multicolumn{5}{|c|}{ Consumo diario total $(\mathrm{WH} /$ día $)=(100+15)$} & 115 \\
\hline & \multicolumn{5}{|c|}{ Máxima demanda de carga instalada (KWH) } & 0.030 \\
\hline
\end{tabular}

Como se muestra en la tabla 1 , se ha empleado una bomba de agua de 12 VDC/ 20 Watts de potencia y dos bombillos LED de 5 Watts cada uno. Esta metodología sugiere añadir un $15 \%$ de pérdidas en el cálculo de la potencia consumida por el sistema en un lapso de 4 horas y 2 horas respectivamente, dando como resultado un consumo diario de $115 \mathrm{WH}$ por día.

\subsection{Cálculos de la energía fotovoltaica}

Para el cálculo de la energía fotovoltaica, se requiere primeramente obtener el rendimiento $\mathrm{R}$; es decir el rendimiento global de la instalación fotovoltaica. Este valor se define como:

$$
\begin{aligned}
& \mathbf{R}=(\mathbf{1}-\mathbf{K b}-\mathbf{K c}-\mathbf{K v}) *(\mathbf{1}-(\mathbf{K a} * \mathbf{N}) / \mathbf{P d}) \\
& \mathrm{R}=(1-0.05-0.1-0.1) *(1-(0.005 * 2) / 0.8) \\
& \mathbf{R}=\mathbf{0 . 7 4} \\
& \mathbf{E f v}=\mathrm{Etfv} / \mathrm{R} \\
& \mathbf{E f v}=115 \mathrm{WH} \text { día/ } 0.74 \\
& \mathbf{E f v}=155.4 \mathrm{WH} .
\end{aligned}
$$

La radiación solar promedio en Panamá por año es de 3.38 $\mathrm{kW} / \mathrm{m} 2 /$ día según [6].

\section{Especificaciones del panel solar}

En la tabla 2, se presenta las especificaciones de un panel solar considerando que suministrará energía para una carga eléctrica pequeña.

Tabla 2. Especificaciones del Panel Solar

\begin{tabular}{|c|c|}
\hline \multicolumn{2}{|c|}{ Especificaciones del Panel Solar } \\
\hline Potencia & 50 Watts \\
\hline Voltaje de circuito abierto & $22.6 \mathrm{~V}$ \\
\hline Corriente de corto circuito & $3.14 \mathrm{~A}$ \\
\hline Voltaje a máxima potencia & $17.5 \mathrm{~V}$ \\
\hline Corriente a máxima potencia & 2.85 \\
\hline
\end{tabular}

\subsection{Determinación del número de paneles}

${ }^{\#} \mathbf{P}=\mathbf{E f v} / \mathbf{W} \mathbf{p}^{*} \mathbf{H}$.

$$
\# \mathrm{P}=155.4 /(50 * 3.38)=\mathbf{1} \text { panel }
$$

\subsection{Determinación del controlador de carga}

Capacidad de corriente del controlador de carga

$$
=(3.14 \mathrm{~A} * 1) * 1.25=3.9 \mathrm{~A}
$$

\subsection{Dimensionamiento de la batería}

Con la tabla 3 es posible determinar la cantidad de baterías necesarias para el sistema solar a implementar. Por su 
López (et al): Validación de un diseño fotovoltaico con un consumo de 30 Watts DC, para ser utilizado con un sistema NFT en comunidades sin acceso a la red eléctrica

capacidad de Amperios/Hora (AH) y su voltaje de operación la batería tiene una capacidad de almacenar $396 \mathrm{WH}$.

Tabla 3. Características de la batería

\begin{tabular}{|l|c|}
\hline \multicolumn{2}{|c|}{ Características de la batería } \\
\hline Voltaje de operación (V) & $12 \mathrm{~V} \mathrm{DC}$ \\
\hline Capacidad de carga (AH) & $33 \mathrm{AH}$ \\
\hline
\end{tabular}

El número de batería se define de la siguiente manera:

155.4 WH/396 WH = 0.4. Dado que las baterías tienen una capacidad de descarga de $50 \%$, entonces su almacenamiento útil es de:

$$
\begin{aligned}
& 336 \mathrm{WH} * 50 \%=168 \mathrm{WH} . \\
& 155.4 \mathrm{WH} / 168 \mathrm{WH}=0.925 .
\end{aligned}
$$

Es decir que se necesita una batería de $33 \mathrm{AH}$ para el sistema empleado.

\section{Proceso de instalación}

Ubicado en un espacio a cielo abierto, se dimensiona el área que ocupa el sistema hidropónico de tipo NFT. Se montan dos tramos de 5 pies de tubería PVC de 4". Cada tramo cuenta con orificios para las plantas de $5.2 \mathrm{~cm}$ de diámetro. Las bases se fabrican con tubos PVC $3 / 4$ ".

La estructura de soporte para tanques de llenado y panel fotovoltaico está confeccionada con hierro negro y tubo galvanizado. La altura mínima y máxima del tanque de llenado es de $1.60 \mathrm{~m}$ y $3.50 \mathrm{~m}$ respectivamente. Se eligió el método de paso de agua con llave sencilla PVC 1/2" para unir ambos tanques de 5 galones, que hace que el líquido mantenga igual nivel en ambos recipientes.

El controlador de carga y la batería se alojan dentro de una caja metálica lejos de la humedad y accesible al operador a una altura de $1.20 \mathrm{~m}$. En figura 1, se muestra un esquema completo en 3D del sistema hidropónico y el sistema solar descrito.

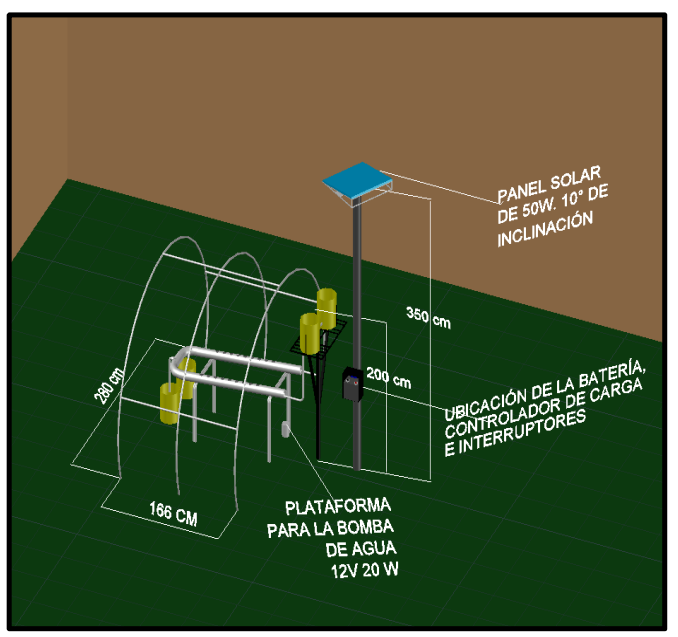

Figura 1. Imagen que muestra el diseño 3D del sistema hidropónico NFT y el sistema solar, creado en AUTOCAD.

\section{Mediciones y resultados}

Luego de haber conectado dispositivos y equipos, se obtienen los siguientes resultados, de acuerdo a las especificaciones técnicas del sistema.

Para la tabla 4, P.N es (parcialmente nublado), S (soleado), $\mathrm{N}$ (nublado), ICC (corriente en corto circuito), VCA (voltaje en circuito abierto). De acuerdo con lo que observa, en el primer día de prueba no se realizó la prueba de corto circuito, ya que no se contaba con información relacionada a la medición en corto circuito del panel solar utilizado.

Tabla 4. Tabla de mediciones relacionada al sistema solar

\begin{tabular}{|l|l|l|l|l|l|}
\hline \multicolumn{7}{|c|}{ Tabla de Mediciones } \\
\hline Fecha & Hora & $\begin{array}{l}\text { ICC } \\
(\mathrm{A})\end{array}$ & $\begin{array}{l}\text { VCA } \\
(\mathrm{V})\end{array}$ & $\begin{array}{l}\text { Radiación } \\
\left(\mathrm{W} / \mathrm{m}^{2}\right)\end{array}$ & Condición \\
\hline \multirow{3}{*}{$12 / 6 / 2018$} & $1: 34$ & & 19.4 & 604 & $\mathrm{P} . \mathrm{N}$ \\
\cline { 2 - 6 } & $1: 54$ & & 19.0 & 415 & $\mathrm{P} . \mathrm{N}$ \\
\hline \multirow{3}{*}{$13 / 6 / 2018$} & $12: 33$ & 4.3 & 20.1 & 1335 & $\mathrm{~S}$ \\
\cline { 2 - 6 } & $1: 00$ & 4.4 & 20.4 & 1390 & $\mathrm{~S}$ \\
\cline { 2 - 6 } & $1: 17$ & 1.2 & 18.9 & 156 & $\mathrm{~N}$ \\
\hline \multirow{5}{*}{$14 / 6 / 2018$} & $11: 33$ & 1.8 & 19.7 & 320 & $\mathrm{~N}$ \\
\cline { 2 - 6 } & $11: 49$ & 2.4 & 19.3 & 336 & $\mathrm{~N}$ \\
\cline { 2 - 6 } & $12: 09$ & 1.9 & 19.4 & 363 & $\mathrm{~N}$ \\
\cline { 2 - 6 } & $12: 52$ & 1.8 & 19.7 & 333 & $\mathrm{~N}$ \\
\cline { 2 - 6 } & $1: 09$ & 1.7 & 19.6 & 301 & $\mathrm{~N}$ \\
\hline
\end{tabular}

Para obtener los datos de radicación se empleó un medidor de potencia solar, como el mostrado en la figura 2.

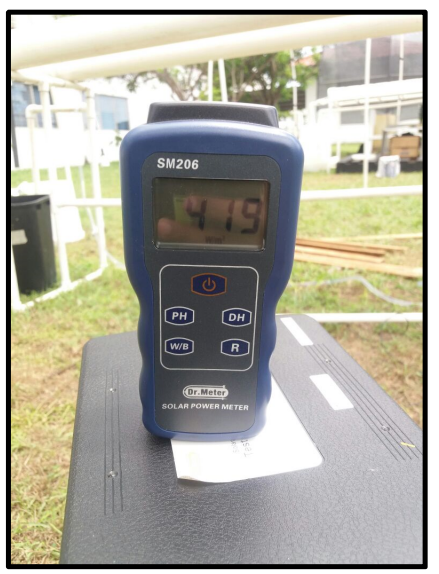

Figura 2. Imagen que muestra la mediación de la radiación solar de $419 \mathrm{~W} / \mathrm{m}^{2}$, utilizando un medidor de potencia solar Dr. Meter, modelo SM206.

En la tabla 5, se muestran los datos de autonomía obtenidos para el sistema solar implementado. Se realizaron diferentes pruebas para comprobar la autonomía del sistema llevándolo a su máximo estrés. Durante las pruebas se hicieron cambios en la configuración del controlador de carga para descargar la batería hasta los $9 \mathrm{~V}$ y cargarla hasta los $14 \mathrm{~V}$. Luego de realizados los cambios se logró un máximo rendimiento de 5 horas con 20 minutos sin recibir carga del panel solar. 
Tabla 5. Prueba de Autonomía

\begin{tabular}{|c|c|c|c|c|c|}
\hline $\begin{array}{c}\text { Estado del } \\
\text { panel } \\
\text { durante la } \\
\text { prueba }\end{array}$ & Fecha & Hora & $\begin{array}{c}\text { Voltaje } \\
\text { antes } \\
\text { del } \\
\text { ciclo }\end{array}$ & $\begin{array}{c}\text { Duración } \\
\text { del ciclo }\end{array}$ & $\begin{array}{c}\text { Voltaje } \\
\text { luego } \\
\text { del } \\
\text { Ciclo }\end{array}$ \\
\hline Desconectado & $12 / 9 / 2018$ & $13: 40$ & $12.9 \mathrm{~V}$ & $2 \mathrm{H}$ & $10.2 \mathrm{~V}$ \\
\hline Desconectado & $12 / 9 / 2018$ & $16: 54$ & $12.7 \mathrm{~V}$ & $40 \mathrm{Min}$ & $10.0 \mathrm{~V}$ \\
\hline Conectado & $13 / 9 / 2018$ & $9: 00$ & $13.7 \mathrm{~V}$ & $2 \mathrm{H}$ & $13.7 \mathrm{~V}$ \\
\hline Desconectado & $13 / 9 / 2018$ & $17: 00$ & $13.7 \mathrm{~V}$ & $3 \mathrm{H}$ & $10.7 \mathrm{~V}$ \\
\hline Desconectado & $17 / 9 / 2018$ & $17: 00$ & $13.7 \mathrm{~V}$ & $2 \mathrm{H}$ & $9.0 \mathrm{~V}$ \\
\hline Desconectado & $18 / 9 / 2018$ & $9: 00$ & $12.2 \mathrm{~V}$ & $1 \mathrm{H} 50 \mathrm{M}$ & $9.3 \mathrm{~V}$ \\
\hline Desconectado & $18 / 9 / 2018$ & $17: 00$ & $13.5 \mathrm{~V}$ & $20 \mathrm{Min}$ & $9.0 \mathrm{~V}$ \\
\hline Desconectado & $19 / 9 / 2018$ & $16: 30$ & $14.0 \mathrm{~V}$ & $4 \mathrm{H}$ & $12.4 \mathrm{~V}$ \\
\hline Desconectado & $20 / 9 / 2018$ & $9: 00$ & $12.3 \mathrm{~V}$ & $1 \mathrm{H} 20 \mathrm{M}$ & $9.2 \mathrm{~V}$ \\
\hline Desconectado & $20 / 9 / 2018$ & $17: 00$ & $12.9 \mathrm{~V}$ & $3 \mathrm{H}$ & $9.0 \mathrm{~V}$ \\
\hline Conectado & $21 / 9 / 2018$ & $10: 00$ & $12.6 \mathrm{~V}$ & $2 \mathrm{H}$ & $12.6 \mathrm{~V}$ \\
\hline Desconectado & $21 / 9 / 2018$ & $14: 00$ & $14.2 \mathrm{~V}$ & $2 \mathrm{H}$ & $12.5 \mathrm{~V}$ \\
\hline
\end{tabular}

\section{Conclusiones}

Basado en pruebas realizadas al sistema NFT y al sistema fotovoltaico, y los resultados obtenidos, concluimos que:

- Es posible utilizar un sistema hidropónico tipo NFT en áreas rurales sin servicio eléctrico; sin embargo, para cultivar mayor número de plantas y obtener mayor variedad de producto saludable las dimensiones del sistema tienen que ser más grandes. Con una bomba $20 \mathrm{~W}$ el sistema solo puede ser aprovechado por familias de pocos integrantes y no para una comunidad completa.

- El panel fotovoltaico de 50w genera suficiente energía para cargar la batería de $33 \mathrm{Ah}$; sin embargo, la misma no mostró tener la capacidad de autonomía de dos días como fue previsto.

- Al momento de elegir una batería para un sistema solar fotovoltaico, es importante tener una referencia de la marca de la batería y su calidad, ya que las baterías de gel son capaces de descargarse al $50 \%$ pero las de mala calidad no logran este rendimiento.

- Es necesario que el equipo de control y almacenamiento de energía (controlador de voltaje y batería) deben tener contacto eléctrico efectivo y alojarse en un lugar lo más seco posible, periódicamente revisar bornes de batería y reaprietes de tornillos de contacto, considerando que es un equipo que está expuesto a la humedad.

\section{AGRADECIMIENTO}

Importante mencionar el apoyo recibido por parte de nuestra institución educativa, al proveer parte de los materiales y el espacio físico para la implementación de este proyecto.

\section{REFERENCIAS}

[1] A.C. García y J.B. Torres, "Diseño y Control de un invernadero de cultivo hidropónico autosuficiente," Universidad Politécnica de Cataluña. [En línea]. Disponible en:
https://upcommons.upc.edu/bitstream/handle/2099.1/17543/M em\%C3\%B2ria.pdf?sequence $=1 \&$ isAllowed $=\mathrm{y}$

[2] A. C. Guevara López, "Diseño E Implementación De Un Sistema De Riego Automatizado, Alimentado Por Un Sistema Fotovoltaico Para El Cultivo Hidropónico En Una Planta Piloto En El Sector De San Vicente-Quero" [En línea]. Disponible en: http://dspace.espoch.edu.ec/handle/123456789/4915

[3] Hydro-Environment, NFT (Nutrient Film Technique). [En línea]. Disponible en: https://www.hydroenv.com.mx/catalogo/index.php?main_page $=$ page $\&$ id $=101$.

[4] C. V. Urrutia, "Plan Energético Nacional 2015-2050. Secretaría Nacional de Energía" 2017. [En línea]. Disponible en: http://www.energia.gob.pa/energia/wpcontent/uploads/sites/2/2017/06/Plan-Energetico-Nacional2015-2050.pdf.

[5] L. Mogollón, (Presentación en clase del tema: Dimensionamiento de un Sistema fotovoltaico, noviembre 2014). Maestría en Energía Renovable, Universidad Tecnológica de Panamá.

[6] Empresa de Transmisión Eléctrica S.A. "Radiación Solar en Panamá, Brillo Solar". [En línea]. Disponible en: https://www.hidromet.com.pa/brillo_solar.php. 